\title{
Historical and Clinical Perspectives of the Expanded Disability Status Scale
}

\author{
John F. Kurtzke \\ Neurology, Georgetown University, and Neurology and Neuroepidemiology, Veterans Affairs Medical Center, \\ Washington, D.C., USA
}

\author{
Key Words \\ Multiple sclerosis - Neurologic signs - Rating scales • \\ Natural history $\cdot$ Treatment trials
}

\begin{abstract}
Background: The measurement of neurologic impairment in multiple sclerosis (MS) is of importance in treatment trials and course of illness. Methods: This review describes the rationale underlying the formation and use of a bifid rating system, the (Expanded) Disability Status Scale (DSS) and the Functional Systems (FS). Results: All signs found at neurologic examination in MS can be consolidated into 8 mutually exclusive FS: pyramidal, cerebellar, brain stem, sensory, bowel and bladder, visual, cerebral and other, each of which, save 'other', is given ordinal grades from 0 to 5 or 6 . Each FS correlates with the DSS, a step scale from 0 (normal) to 10 (death due to MS), which is an overall measure of neurologic abnormality. Dividing steps 1-9 each into 2 gives the Expanded DSS. The FS and DSS were used in an Army series to describe neurologic status at first diagnosis and over the first 20 years of illness. DSS severity at 5 years after onset, but not earlier, was highly predictive of later severity. Conclusions: Combination of the (Expanded) DSS and FS has been used successfully to assess impairment for natural history and treatment studies in MS.

Copyright $\odot 2008$ S. Karger AG, Basel
\end{abstract}

(C) 2008 S. Karger AG, Basel

Accessible online at:

www.karger.com/ned

\section{Introduction}

If one person could be considered the founder of modern clinical neurology, it would be Jean-Martin Charcot. His lectures at La Salpêtrière on diseases of the nervous system were given from 1866 to 1880 [1]. Among the illustrations in his text was one of the spinal cord in multiple sclerosis (MS). This showed clearly a high proportion of white matter involved by these lesions, which were indeed 'scattered in time and space' throughout the cord from one end to the other, and including as much as an entire cross-section. It also showed an absence of gross wallerian degeneration, despite such a degree of involvement. There were similar lesions in the brain stem, while the proportion of cerebral white matter thus affected was much lower.

The principal clinical deficits in MS would then be expected to be the result of lesions of the spinal cord and brain stem, with multiple lesions along the neural pathways required for impairment of function. In this manner, the involvement of long tracts would give predominant impairment in the lower extremities, and bilaterally so.

Presented at the 21st Annual Meeting of the Consortium of Multiple Sclerosis Centers (Clinical Course No. 4), Washington, May 30 to June $2,2007$.

\section{KARGER \\ Fax +4161306 12}

E-Mail karger@k.karger.com
Prof. John F. Kurtzke, MD, Emeritus of Neurology

7509 Salem Road

Falls Church, VA 22043-3240 (USA)

Tel. +1 703560 6016, Fax +1 7035606490

E-Mail kurtzke2@aol.com 
Table 1. Patterns of limb involvement (\%) in MS for motor signs and symptoms, coordination and sensory symptoms, and sensory signs by type

\begin{tabular}{|c|c|c|c|c|c|c|c|}
\hline \multirow{2}{*}{$\begin{array}{l}\text { Limbs } \\
\text { involved }\end{array}$} & \multicolumn{2}{|c|}{ Motor } & \multirow{2}{*}{$\begin{array}{l}\text { Coord. } \\
\text { symptoms }\end{array}$} & \multirow{2}{*}{$\begin{array}{l}\text { Sensory } \\
\text { symptoms }\end{array}$} & \multicolumn{3}{|c|}{ Sensory signs } \\
\hline & signs & symptoms & & & $\mathrm{tc}, \mathrm{pn}$ & posit. & vibr. \\
\hline None & 36.1 & 37.7 & 50.1 & 62.5 & 62.5 & 49.9 & 36.8 \\
\hline $1(\mathrm{U})$ & 1.2 & 3.6 & 2.6 & 5.0 & rest & 0 & 0 \\
\hline $1(\mathrm{~L})$ & 5.2 & 7.8 & 1.9 & 4.6 & rest & 0 & 0 \\
\hline $2(\mathrm{U})$ & 0.2 & 0.9 & 3.9 & 4.3 & 2.0 & 3.0 & 4.1 \\
\hline $2(\mathrm{~L})$ & 30.2 & 22.8 & 25.4 & 8.6 & 24.9 & 30.9 & 37.4 \\
\hline $2(\mathrm{U}, \mathrm{L})$ & 3.6 & 9.7 & 1.7 & 5.9 & rest & 0 & 0 \\
\hline $3(1 \mathrm{U}, 2 \mathrm{~L})$ & 11.0 & 4.0 & 2.3 & 3.4 & rest & 0 & 0 \\
\hline 4 & 12.5 & 13.4 & 12.0 & 6.4 & 3.5 & 16.3 & 21.8 \\
\hline Total & 100.0 & 100.0 & 100.0 & 100.0 & 100.0 & 100.1 & 100.1 \\
\hline Number & 582 & 2,019 & 2,019 & 2,019 & 541 & 541 & 541 \\
\hline
\end{tabular}

Signs: data of Mueller 1949 [2]; symptoms: data of Kurtzke 1970 [3].

Rest $=$ Total of $7.1 \%$ for these four patterns. Table adapted from Kurtzke [3, 4]. U = Upper limb; $L=$ lower limb; coord. = coordination; $\mathrm{tc}=$ touch; $\mathrm{pn}=$ pain; posit. $=$ position; vibr. $=$ vibration .

\section{Clinical Involvement by Limbs Affected}

In fact, such are the findings in MS, as demonstrated by the frequencies for patterns of limbs involved for the signs among the patients of Ragnar Mueller's monograph on MS throughout Sweden [2], and those for the symptoms $[3,4]$ found for cases from a World War II US Army series, considered further below.

The motor signs and symptoms, the sensory signs and the coordination symptoms were all much more common in the lower limbs, and were most often bilateral (table 1). The most common patterns for abnormalities in these 3 modalities were both lower limbs affected in $25-37 \%$ of all cases, and then all 4 limbs in $13-22 \%$. No involvement was recorded for some feature of these 3 in $36-63 \%$. Cerebellar system limb ataxia would then seem to be due to spinal cord lesions rather than those at higher levels. Sensory symptoms however behaved differently, with no limb predilection found among the 7 affected limb patterns observed (3/5 of patients had none). These symptoms likely reflect lesions of the dorsal root entry zone as the basis for the peculiar 'numbness' of which so many patients complain.

\section{Clinical Diagnostic Criteria}

While the primary focus of this paper is on one method of measuring impairment in MS, some attention needs be paid to the entity that is being measured. An opportunity to explore both the early clinical features of this disease and its definition was provided by a unique series of white men with final diagnoses of MS in US Army hospitals during World War II [5]. We had excluded the small numbers of blacks and women to avoid possible bias in our epidemiologic assessment of the series. Such a diagnosis was reason for medical discharge from service, and the records were therefore unusually complete. Further, postservice examinations were the basis for disability ratings and were also quite detailed. In the early 1960s, we reviewed all their available Army, Veterans Administration (VA) and private medical records. For those with no recent information, we sought examinations performed especially for this study by neurologists across the country. This resulted in nearly 3,000 neurologic examinations covering the first 20 years or so of illness among the 762 men diagnosed as having MS in the Army. For each examination, symptoms were recorded by type and body part affected, as already noted, and the signs were coded to the Disability Status Scale (DSS) and its 8 Functional Systems (FS; see below) [6].

Utilizing all these materials, the 4 neurologists of our study all agreed as to the correct diagnosis for each pa- 
tient. We judged 527 men to be cases of MS: 476 definite and 51 probable. There were also 146 we had classed as definitely 'not MS' (other specific diagnoses) [5].

The diagnostic criteria used were those later proposed by the Schumacher Panel [7], which I think are still the best and the simplest set for the clinical diagnosis of MS. All of the strictly clinical criteria of the Poser Committee [8] met the Schumacher requirements. This is true as well for the more recent diagnostic recommendations of the McDonald International Panel published in 2001 [9], but perhaps less so for the 2005 revision [10].

\section{Validation of Diagnostic Criteria}

Validation of our criteria was made possible with the findings in this Army series by comparisons between the 527 diagnosed MS cases versus the 146 we had agreed had had specific disorders other than MS. Clinically, as one might expect, there was little difference in the neurologic examination at the time of the Army diagnosis. For our epidemiologic study of this series, each of the 762 men had been matched with a military peer on age, sex, race, date of service entry and survival of the war. This provided an unbiased, nationwide, preillness case-control comparison of early MS [11] which served to assess potential risk factors for the MS versus their controls, and to see whether any such factors found were specific to the disease or were merely a marker of neurologic impairment in general. All the case-control comparisons showed major differences between the MS group and its controls as well as the non-MS group and its own controls. The MS had a strong positive relationship with the level of education before service, from an MS/control ratio of 0.73 for 8 years or less steadily up to 1.71 for $\geq 13$ years. The nonMS group showed no gradient. The Army's IQ measure, the General Classification Test, given at service entry provided a similar dichotomy. The higher the score, the greater the risk of MS, while there was no such trend among the non-MS cases. The socioeconomic status of preservice occupation as scored by the Bureau of Census also had a strong positive correlation with the MS cases, increasing from a 0.75 ratio for the lowest quintile to 1.61 for the highest, but with none for the non-MS group. Urbanization of preservice residence also provided this same differentiation, with an MS/control ratio ranging from 2.32 for the most urban down to 0.62 for the rural residence. No gradient was seen for the non-MS/control comparisons.

Perspectives of the EDSS
There was also a north-south gradient of risk by tier of residence at entry into service, which also illustrates the value of our diagnostic review. For the MS, the MS/control ratios were 1.39 north, 0.86 middle and 0.80 south $(p<0.01)$. The ratios for the non-MS cases versus their own controls were 0.63 north, 1.11 middle and 1.33 south $(p>0.10)$. Combining these two subsets to approximate the original Army series provided respective case/control ratios of 1.19, 0.91 and 0.93 ( $\mathrm{p}>0.20$ ), and geography was then no longer a significant risk factor.

Thus, in every testable aspect, the subset of the Army series that we had called MS defined a distinct group with case-control differences which were not seen for the nonMS cases from the same series versus their own controls. Therefore the diagnostic criteria we used appeared to have considerable external validation and not only clinical consensus, and their use was critical in assessing risk factors.

\section{FS at First Diagnosis}

All these patients were free of neurologic impairment before the Army diagnostic bout. For the 335 men of the 527 MS cases whose examination was complete enough to grade fully all $8 \mathrm{FS}$, more than 4 out of 5 then had pyramidal tract involvement (proportion affected $=0.82$ ). Cerebellar $(\mathrm{p}=0.77)$, brain stem $(\mathrm{p}=0.76)$ and sensory systems $(p=0.50)$ were the other FS most often affected. These 4 were classed as the major FS, with abnormalities in each one in the majority of patients, even at this very early stage of clinical disease. The 4 minor FS, much less often affected, were bowel and bladder (at 0.20 involved), visual (0.35), cerebral (0.21) and other (0.13). Together, these $8 \mathrm{FS}$ - which are all mutually exclusive - include all deficits seen in this disease that can be defined at neurologic examination $[12,13]$.

\section{FS Patterns at First Diagnosis}

With this exclusivity and completeness, and by keeping the FS in this constant order, each patient's neurologic status can then be described qualitatively on a yes-no basis with an 8-digit binary number, with a ' 1 ' for involvement in a given system, regardless of severity and a ' 0 ' for no involvement. For example, ' 11 ' followed by 6 zeroes would describe a patient with only pyramidal and cerebellar signs. There are then a total of 256 possible patterns ( 2 to the 8 th power) into which every patient must fall.

Neuroepidemiology 2008;31:1-9 
Table 2. Numbers of patients showing specific patterns of involvement (1) or no involvement (0) within each of 8 FS at first Army diagnosis, US Army World War II hospital series (modified from Kurtzke [13])

\begin{tabular}{|c|c|c|c|c|c|c|c|c|c|c|c|c|c|c|c|c|c|}
\hline & \multicolumn{16}{|c|}{ Minor FS } & \multirow{2}{*}{$\begin{array}{l}\text { Major } \\
\text { total }\end{array}$} \\
\hline & 0000 & $\begin{array}{l}1000 \\
\text { (BB) }\end{array}$ & $\begin{array}{l}0100 \\
(V)\end{array}$ & $\begin{array}{l}0010 \\
(\mathrm{Cb})\end{array}$ & $\begin{array}{l}0001 \\
(\mathrm{O})\end{array}$ & 1100 & 1010 & 1001 & 0101 & 0110 & 0011 & 1110 & 1101 & 1011 & 0111 & 1111 & \\
\hline \multicolumn{18}{|l|}{ Major FS } \\
\hline 0000 & - & 1 & 3 & - & - & - & - & - & - & - & - & - & - & - & - & - & 4 \\
\hline $1000(\mathrm{P})$ & 3 & 3 & 1 & - & 1 & - & - & - & - & - & - & - & - & - & - & - & 8 \\
\hline 0100 (Cll) & 1 & - & 1 & 1 & - & 1 & - & - & 1 & - & - & - & - & - & - & - & 5 \\
\hline 0010 (BS) & 6 & - & 9 & - & 1 & - & - & - & - & - & - & - & - & - & - & - & 16 \\
\hline $0001(S)$ & - & - & 1 & - & - & - & 1 & - & - & - & - & - & - & - & - & - & 2 \\
\hline 1100 & 8 & 2 & 2 & 1 & 1 & - & - & 1 & - & 2 & - & - & - & - & - & - & 17 \\
\hline 1010 & 7 & - & 5 & 1 & 1 & 1 & - & - & - & - & 1 & - & - & - & - & - & 16 \\
\hline 1001 & 5 & 2 & 3 & 2 & 2 & 2 & - & 1 & - & - & - & - & - & - & - & - & 17 \\
\hline 0101 & - & - & - & - & 1 & - & - & - & - & - & - & - & - & - & - & - & 1 \\
\hline 0110 & 9 & 1 & 5 & 1 & 1 & 1 & - & - & - & 3 & 1 & - & - & - & - & - & 22 \\
\hline 0011 & 1 & - & 1 & - & - & - & - & - & - & - & - & - & - & - & - & - & 2 \\
\hline 1110 & 29 & 4 & 16 & 8 & 3 & 4 & 2 & - & 2 & 6 & - & 1 & - & 3 & - & - & 78 \\
\hline 1101 & 14 & 3 & 2 & 2 & 2 & 1 & - & 2 & - & - & - & 1 & - & - & - & - & 27 \\
\hline 1011 & 6 & - & 3 & - & 1 & 1 & - & - & - & 1 & - & - & - & - & - & - & 12 \\
\hline 0111 & 1 & - & 1 & 1 & - & 1 & - & - & 1 & - & - & - & - & - & 1 & - & 6 \\
\hline 1111 & 31 & 11 & 12 & 15 & 7 & 5 & 2 & 2 & 2 & 6 & 1 & 4 & 1 & 1 & 1 & - & 101 \\
\hline Minor total & 121 & 27 & 65 & 32 & 21 & 17 & 5 & 6 & 6 & 19 & 3 & 6 & 1 & 4 & 2 & - & 335 \\
\hline
\end{tabular}

$\mathrm{P}=$ Pyramidal; $\mathrm{Cll}=$ cerebellar $; \mathrm{BS}=$ brain stem $\mathrm{S}=$ sensory $\mathrm{BB}=$ bowel and bladder $\mathrm{V}=$ visual; $\mathrm{Cb}=$ cerebral; $\mathrm{O}=$ other.

The individual patterns observed for the entire series can then be shown as an X-Y plot with the 4 minor FS listed in order [0000, 1(BB)000, 01(V)00, 001(Cb)0, $0001(\mathrm{O})]$; then $1100,1010,1001,0101,0110,0011$; and 1110 , $1101,1011,1111]$, all providing the headings for the columns, and the 4 major FS in similar order $[0000,1(\mathrm{P}) 000$, 01(Cll)00, 001(BS)0, 0001(S)..., 1111] giving the headings for the rows (table 2). The letters in parentheses and in the table headings for single-system involvement indicate to which specific FS the ' 1 ' belongs. The 8 patients with only pyramidal and cerebellar signs would then occupy the 6th row (1100) of the first column (0000). A high proportion of patients showed multiple involvement in the $4 \mathrm{ma}-$ jor FS even at first diagnosis, with only a paucity of multiple involvements in the 4 minor FS.

Now if occurrence of disease affecting one system is independent of involvement in any other system, then a specific frequency expected for each of the 256 patterns can be calculated as the product of the 8 observed frequencies in the total series (table 3). For involvement of only pyramidal and cerebellar systems, this expected frequency would be $0.82 \times 0.77$ (the ' $1 \mathrm{~s}$ ') times all the 0 s: $0.24 \times 0.50 \times 0.80 \times 0.65 \times 0.79 \times 0.87$. This product is 0.028 , which would mean an expectation of 9.3 cases for this specific pattern among the 335 patients. The observed number was 8 . This was the sixth most common pattern expected. Fourteen specific patterns were expected to constitute half of all the cases, and they did (table 4). These patterns showed a marked preponderance of multiple involvement among the 4 major FS. Ninety percent of patients were expected to fall within the 86 most common patterns, and $90 \%$ were observed. The frequency of the specific patterns observed and expected was virtually identical for each one of these 86 .

\section{The Need for an (Expanded) DSS or Its Equivalent}

The FS, though, are of limited value in describing patterns of neurologic involvement when each system is quantified. There would then be over one million possible patterns into which patients might fall. Adding scores for the separate FS to provide a single total score to me is not valid, as these are not arithmetic scales. This would be apparent if the grades were labeled 'a' to ' $\mathrm{g}$ ' instead of ' 0 ' to ' 6 ' and an average of ' $c a / b$ ' were presented. I realize 
Table 3. Frequency of involvement by FS at first Army diagnosis, US Army World War II hospital series

\begin{tabular}{lrrrl}
\hline \multirow{2}{*}{ FS } & \multicolumn{3}{l}{ Involvement } & \\
\cline { 2 - 5 } & yes (1) & no $(0)$ & $\mathrm{p}(1)$ & $\mathrm{p}(0)$ \\
\hline Major & & & & \\
$\quad$ Pyramidal & 270 & 59 & 0.82388 & 0.17612 \\
$\quad$ Cerebellar & 257 & 78 & 0.76716 & 0.23284 \\
Brain stem & 253 & 82 & 0.75522 & 0.24478 \\
$\quad$ Sensory & 168 & 167 & 0.50419 & 0.49651 \\
Minor & & & & \\
$\quad$ Bowel and bladder & 66 & 269 & 0.19701 & 0.80299 \\
$\quad$ Visual (neuropathic signs) & 116 & 219 & 0.34627 & 0.65353 \\
$\quad$ Cerebral (includes grade 1) & 71 & 264 & 0.21194 & 0.78806 \\
$\quad$ Other & 43 & 292 & 0.12836 & 0.87164 \\
\end{tabular}

$\mathrm{p}(1)=$ Proportion 'yes'; $\mathrm{p}(0)=$ proportion 'no'. Modified from table 2 of Kurtzke [13].

that such a quibble is perhaps not often accepted at present, and parametric assessments of clearly nonparametric scales are quite widespread. But I was taught that numbers are operationally adjectives and not nouns, and thus ask myself ' 3 what' for a grade on a scale. That such calculations often 'look good' (e.g. Likert scales) may not be a justification.

In general, scales can be divided into 4 types: nominal (yes, no; male, female); ordinal or rank order $(a>b>c)$; interval with equal divisions between steps (temperature); and ratio (one step $4=$ two step 2; one step $1+$ one step 3 = average of step 2). I was taught that only the last two were appropriate for parametric tests. In addition, as noted below, the grades on one FS scale are not equivalent to those in another: they show notably different frequency distributions, not only among the scales, but also by severity of involvement within each scale $[3,4]$. I had early concluded that another approach was needed to provide a single figure to estimate the severity of total neurologic involvement in each patient. And such an overall measure of neurologic impairment was therefore devised, the DSS, which had steps from 0 (normal neurologic examination) to 10 (death due to MS), and each step with its defining approximate equivalents in FS scores (table 5) [14]. The DSS later evolved into the Expanded DSS (EDSS) by dividing into 2 each step from DSS 1 to 9, and retaining FS equivalents for each step [15]. For example, patients graded 2.0 plus those 2.5 on the EDSS would together comprise those graded 2 on the DSS.
Table 4. Patterns of involvement by FS at first Army diagnosis, US Army World War II hospital series, ranked by frequency of number of expected cases, compared with number of cases observed, for all patterns where expected cases $\geq 5.0$

\begin{tabular}{clcc}
\hline \multirow{2}{*}{ Rank } & Pattern & \multicolumn{2}{l}{ Cases } \\
\cline { 3 - 4 } & & observed & expected \\
\hline 1 & 11110000 & 31 & 28.92 \\
2 & 11100000 & 29 & 28.74 \\
3 & 11110100 & 12 & 15.32 \\
4 & 11100100 & 16 & 15.23 \\
5 & 11010000 & 14 & 9.37 \\
6 & 11000000 & 8 & 9.32 \\
7 & 10110000 & 6 & 8.78 \\
8 & 10100000 & 7 & 8.72 \\
9 & 11110010 & 15 & 7.78 \\
10 & 11100010 & 8 & 7.73 \\
11 & 11111000 & 11 & 7.09 \\
12 & 11101000 & 4 & 7.05 \\
13 & 01110000 & 1 & 6.18 \\
14 & 01100000 & 9 & 6.15 \\
$15+$ & all others & 164 & 168.63 \\
\hline 256 & total & 335 & 335.01 \\
\hline
\end{tabular}

$\chi_{14}^{2}=20.58, p>0.10$ for observed versus expected cases. Rank under the hypothesis of independence; pattern: $1=$ involved; $0=$ not involved. Modified from table 2 of Kurtzke [15].

Table 5. Outline of DSS (with approximate FS equivalents in parentheses), as of 1961

\section{DSS Definition}

0 Normal neurologic examination (all FS grade 0 , but cerebral grade 1 included)

1 No disability, minimal signs (any/all FS grade 1)

2 Minimal disability (1 or 2 FS grade 2 )

3 Moderate disability (1 or 2 FS grade 3 or several grade 2 )

4 Disability not preventing normal activities (1 FS grade 4 or several $\leq 3$ )

5 Disability limiting walking to few blocks (1 FS grade 5 or lesser combined)

6 Assistance needed for walking (1 FS grade 6 or lesser combined)

7 Wheelchair but self-moving (FS usually grade 4+ in several)

8 Bed patient but with arm function (FS usually grade $4+$ in many)

9 Totally helpless bed patient (FS usually grade 4+ in most)

10 Death due to MS

Modified from Kurtzke [6], where more complete definitions can be found. 
Table 6. Tschuprow coefficients of correlation for the FS and the DSS for some 1,700 examinations over the first 20+ years of illness in the MS patients of the US Army World War II hospital series

\begin{tabular}{llllllll}
\hline FS & FS & & & & & DSS \\
\cline { 2 - 5 } & Cll & BS & S & BB & Cb & \\
\hline Pyramidal & \multirow{2}{*}{0.32} & 0.24 & 0.22 & 0.35 & 0.12 & 0.50 \\
Cll & & 0.26 & 0.17 & 0.24 & 0.16 & 0.45 \\
BS & & & 0.10 & 0.24 & 0.14 & 0.27 \\
S & & & & 0.14 & $0.04^{1}$ & 0.23 \\
BB & & & & & 0.12 & 0.31 \\
Cb (total) & & & & & & 0.14 \\
\hline
\end{tabular}

$\mathrm{Cll}=$ Cerebellar; $\mathrm{BS}=$ brain stem; $\mathrm{S}=$ sensory; $\mathrm{BB}=$ bowel and bladder; $\mathrm{Cb}=$ cerebral. Modified from table 16 of Kurtzke [4].

${ }^{1}$ Not significant $(\mathrm{p}>0.05)$

\section{Use of FS and DSS in Early Treatment Trials}

The DSS and FS were used, along with other measures, in the first two multicentered, randomized, double-blind, placebo-controlled trials of therapy ever conducted in MS. The first of these was a VA Cooperative Study published in the Journal of the American Medical Association in 1957 [16]. This study was designed to test our earlier claim that isoniazid might be an effective treatment for MS (for which purpose this rating system had been originally devised) [17]. It was not.

The second treatment trial was a cooperative study by 10 university-based MS treatment centers of adrenocorticotrophin in acute exacerbations. Its preliminary report was published as a supplement in Neurology in 1968 [18]. This included the complete protocol of the study as defined in April 1965, with all the study forms used. One of these forms, ms4, was entitled 'Neurologic Status Evaluation' and consisted of the 8 FS and the DSS, with summarized definitions for each step of each system. The final report, also a Neurology supplement, appeared in 1970 [19].

\section{FS Findings and the DSS}

In the Army series, among the 527 MS cases some 1,700 examinations in the first 20 years of illness were scored not only on the FS, but also on the DSS. The frequency distribution overall showed a unimodal and fairly normal curve for DSS steps 0-9, with about $20 \%$ each at DSS 3 and 4 . The frequency of involvement in each FS increased with increasing DSS scores, and the order of involvement, pyramidal more than cerebellar more than brain stem and so on, remained almost the same regardless of DSS [4]. The more severely affected patients (DSS 5+) almost all had pyramidal and cerebellar signs, and three quarters brain stem and sensory signs. Only bowel and bladder function altered the sequence, with then even more frequent involvement for this spinal cord sign than for some others: for DSS 7-9, 80\% had bowel and bladder impairment. Cerebral and other FS were the least commonly involved at any stage of severity.

\section{Interrelations of the FS and the DSS}

Correlations among 6 graded FS and the DSS were assessed with a Tschuprow test, which I was told is a nonparametric measure of association among rank-order scales. These coefficients range from 0 (no correlation) to +1.0 (perfect positive correlation) [4]. The visual system could not be included here because so few of the patients had had formal, mapped-out visual fields recorded, a requirement as the scale had been devised.

There was a significant correlation for all comparisons except cerebral versus sensory (table 6). All correlations were low for cerebral function (0.04-0.16) for reasons noted below. But the correlations were as good or better for all of these with the DSS than with any pair of FS. For the DSS the coefficients were 0.50 (pyramidal), 0.45 (cerebellar), 0.27 (brain stem), 0.23 (sensory), 0.31 (bowel and bladder) and 0.14 (cerebral).

To support these findings more directly, frequency distributions for each FS at the several levels of severity indicated by the DSS were plotted [13]. Both the pyramidal and the cerebellar systems showed marked increases in both frequency and severity with increasing DSS scores. The same relationships but at lower levels were seen for brain stem and sensory systems. Similar increases in both features were present for bowel and bladder. Trends were similar in visual function scores derived from a separate VA hospital series.

Cerebral functions also showed increases in frequency and severity with increasing DSS. Step 1 on the scale referred to mood or behavior changes with otherwise intact mental status and of itself did not contribute to the DSS. Here $79 \%$ of examinations were scored as 0 and $16 \%$ as step 1. For the latter there was no real gradient from the $12 \%$ at DSS 0 to the $17 \%$ at DSS 7-9. This is why the correlations considered above were so low. The true menta- 
tion impairment showed the same gradients as the others. Steps $2+3$ went steadily from less than $1 \%$ for DSS $1-2$ up to $6 \%$ for DSS 7-9. Steps $4+5$ were $2 \%$ at DSS 5-6 and $9 \%$ at DSS 7-9. The proportions with clinical evidence of impaired mentation may seem unusually low, but these frequencies may well be accurate for this series of relatively early MS. Recall too that in this era most academicians and practitioners were neuropsychiatrists, and even 'we few, we happy few, we band of brothers' [Henry V], we 'pure' neurologists, had had solid grounding in psychiatry during training. Therefore the low frequencies reported do not appear to me to be the result of inadequate assessments.

Therefore, overall I think the DSS does seem to reflect quite well the severity of neurologic impairment in MS. And its emphasis on ambulation would seem an asset rather than a drawback. Further, its interrelations with the FS provide the reason, I think, why other scoring systems also seem to work well in MS. So long as they show some gradient as patients worsen, they will appear appropriate. To me, the main question is, which clinical system is 'best' in approximating that which we would all like to know (but don't): how much neural tissue is involved in this disease at this time? I do not think lesion load or other MRI measures really answer this, especially when most of the clinical impairment is the result of (multiple) lesions of the spinal cord and brain stem, as noted earlier.

\section{DSS over Time}

The DSS in the Army series showed a unimodal and rather mild involvement at first diagnosis. A progressive worsening over time was clear, with cumulative frequency crossing $50 \%$ at a point that would be equivalent to a DSS score of 3.2 at diagnosis, and 3.5, 4.1 and 4.7 at 5, 10 and 15 years after onset. However, about 0.25 of the cases still remained mild - DSS 0-2 - throughout this followup interval [20].

Figure 6 of the Handbook of Clinical Neurology chapter cited [3] showed frequency distributions by DSS score for 4 sets of early MS cases (p. 190). The first curve, with a high modal value at 3 for the DSS, described onsets in the subset of the Army patients who had not been diagnosed as having MS when examined at onset, but who had been so labeled at the later diagnostic bout. The next curve, with a high mode of 3-4, reflected those where the Army diagnostic bout was also the onset bout. The third curve, with a low mode at DSS 5 represented scores from VA hospital patients an average of 6 years after onset. These were the same VA patients who had provided the clinical materials used to formulate the FS and the DSS. All of them had had detailed examinations listing all positive and negative findings at both admission to and discharge from the hospital. The last curve showed DSS scores for another VA hospital series with a mean duration of 16 years from onset. There was in this curve the first appearance of bimodality in the distribution, with a low mode at DSS 4 and a high mode at DSS 7. It may be that the bimodal EDSS that so many series show reflects not only an average later stage of illness than these Army and early VA cases, but also a division into 'benign' and 'not benign' MS.

\section{Prognosis from Early Clinical Findings}

The Army series also permitted us to explore prognosis in this disease [21]. Bout frequency early in the illness has been used as one such measure. While there was a slight trend toward worse disease with increasing bouts, it did not appear to be a very strong predictor. At 15 years after diagnosis, the proportions of those who were then mild (DSS 0-2) and severe (DSS 6+) among those who in the first 5 years of illness had had a single (onset) bout were 22 and $41 \%$, respectively, while those with $5+$ bouts in the 5 years were $18 \%$ mild and $65 \%$ severe. In like manner, the severity of neurologic impairment at first diagnosis did not seem too useful either. For mild cases at diagnosis, only 7\% were mild and 57\% severe 15 years later. Respective frequencies for the severe cases were 16 and $72 \%$. Similar were the findings for the onset bout. However, the neurologic status at 5 years after onset was a very strong predictor of the course for the next 13 years. Two thirds of patients with DSS 0-2 at 5 years after onset were still 0-2 15 years after diagnosis, and only $11 \%$ were severe. For the severely involved patients at 5 years, $99 \%$ were severe at the latter point and half were dead. This has been called by some the '5-year rule' for prognosis in MS. A similar prognostic value could be seen from the neurologic examination itself at 5 years after onset. Patients who then had neither pyramidal nor cerebellar signs had almost a 3 -in- 4 chance of being a mild case at the follow-up, and only $5 \%$ were severe. For those with at least moderate involvement in both systems, none were mild and 9 out of 10 were then severe or dead. 


\section{Comment}

The neurologic assessment system consisting of the 8 FS and the (E)DSS has been criticized on a number of bases. Those up to 1989 have been discussed extensively [13]. Later proposed amendments, deletions and alterations have all, in my view, been irrelevant or even counterproductive. I have seen patients fitting the criteria for every step in each FS and the EDSS. Assignment of steps should not be difficult after a careful neurologic examination. In doubtful cases, one should bracket the possibilities and decide which is closer. Recall that the EDSS above 5.0 does require FS involvement as well as gait impairment. Where these two aspects are markedly discrepant, I think the whole neurologic examination should be repeated.

A quotation from a recent paper might be an appropriate way to end this review:

During my residency we assessed a possible treatment for MS, which required a comparison group and a means of measuring change. The former comprised the records of over $300 \mathrm{MS}$ patients, whose neurological deficits were then consolidated into mutually exclusive functional systems, each with grades for severity. As rank-order scales they could not be summed or compared directly, but they were used as the basis for the DSS, which ranged from 0 (normal) to 10 (death due to MS). This scale was later expanded into the EDSS by halving each step 1 through 9. This bifid system is applicable to all patients with MS regardless of type or severity of neurological impairment [22].

\section{Disclosure}

The author is solely responsible for the origin, development, revisions and publications of the (E)DSS and its FS (functional groups) as a method of rating neurologic involvement in multiple sclerosis $[3,4,6,13-15,22,23]$. This system was first called the Neurologic Status Evaluation in the published 1965 protocol included in the preliminary report of the adrenocorticotrophin study of Rose et al. [18].

These works were based on his detailed review and analysis of the clinical records of patients in 3 VA Hospitals, the first that in the Bronx, N.Y., then that in Coatesville, Pa., and lastly that in Washington, D.C. All these activities were performed while the author was a fulltime employee of the Department of Medicine and Surgery in these VA institutions. As such, he was considered 'on duty' and available at all hours and on all days (perhaps the origin of the cliché '24/7'?). Accordingly, the products of this endeavor are the property of the US Federal Government, and, as such, the author had been informed that they could not be copyrighted by any person, institution or organization - at least in the United States. Rather, they are in the public domain available without charge for use by anyone. The author has never received any financial remuneration in any form for the use of these materials.

\section{References}

1 Charcot J-M: Leçons sur les maladies du système nerveux faites à la Salpêtrière, recuillies et publiées par Bourneville, 3e éd. Paris, Delahaye et Cie, 1877, tome ler.

2 Mueller R: Studies on disseminated sclerosis with special reference to symptomatology, course and prognosis. Acta Med Scand 1949; 133(suppl 222):1-214.

3 Kurtzke JF: Clinical manifestations of multiple sclerosis; in Vinken P, Bruyn G (eds): Handbook of Clinical Neurology. Amsterdam, North-Holland, 1970, vol 9: Multiple sclerosis and other demyelinating diseases, pp 161-216.

$\checkmark 4$ Kurtzke JF: Neurologic impairment in multiple sclerosis and the Disability Status Scale. Acta Neurol Scand 1970;46:493-512.

5 Nagler B, Beebe GW, Kurtzke JF, Kurland LT, Auth TL, Nefzger MD: Studies on the natural history of multiple sclerosis. 1. Design and diagnosis. Acta Neurol Scand 1966;42(suppl 19):141-156.
6 Kurtzke JF: On the evaluation of disability in multiple sclerosis. Neurology 1961;11:686694.

7 Schumacher GA, Beebe G, Kibler RF, Kurland LT, Kurtzke JF, McDowell F, Nagler B, Sibley WA, Tourtellotte WW, Willmon TL: Problems of experimental trials of therapy in multiple sclerosis: report by the Panel on the Evaluation of Experimental Trials of Therapy in Multiple Sclerosis. Ann NY Acad Sci 1965;122:552-568

$\checkmark 8$ Poser CM, Paty DW, Scheinberg L, McDonald WI, Davis FA, Ebers GC, Johnson KP, Sibley WA, Silberberg DH, Tourtellotte WW: New diagnostic criteria for multiple sclerosis: guidelines for research protocols. Ann Neurol 1983;13:227-231.
9 McDonald WI, Compston A, Edan G, Goodkin D, Hartung H-P, Lublin FD, McFarland HF, Paty DW, Polman CH, Reingold SC, Sandberg-Wollheim M, Sibley W, Thompson A, van den Noort S, Weinshenker BY, Wolinsky JS: Recommended diagnostic criteria for multiple sclerosis: guidelines from the International Panel on the Diagnosis of Multiple Sclerosis. Ann Neurol 2001;50:121-127.

10 Polman CH, Reingold SC, Edan G, Filippi M, Hartung H-P, Kappos L, Lublin FD, Metz LM, McFarland HF, O'Connor PW, Sandberg-Wollheim M, Thompson AJ, Weinshenker BG, Wolinsky JS: Diagnostic criteria for multiple sclerosis: 2005 revisions to the 'McDonald Criteria'. Ann Neurol 2005;58: 840-846.

11 Beebe GW, Kurtzke JF, Kurland LT, Auth TL, Nagler B: Studies on the natural history of multiple sclerosis. 3. Epidemiologic analysis of the Army experience in World War II. Neurology 1967;17:1-17. 
12 Kurtzke JF, Beebe GW, Nagler B, Auth TL, Kurland LT: Studies on the natural history of multiple sclerosis. 6. Clinical and laboratory findings at first diagnosis. Acta Neurol Scand 1972;48:19-46.

13 Kurtzke JF: The Disability Status Scale for multiple sclerosis: apologia pro DSS sua. Neurology 1989;39:291-302 (available on CD as: Kurtzke JF: EDSS bibliographic material. 21st Annual Meeting of the Consortium of MS Centers, Washington, May 30 to June 2, 2007, support@mscare.org).

14 Kurtzke JF: A new scale for evaluating disability in multiple sclerosis. Neurology 1955; 5:580-583 (available on CD as: Kurtzke JF: EDSS bibliographic material. 21st Annual Meeting of the Consortium of MS Centers, Washington, May 30 to June 2, 2007, support@mscare.org).

15 Kurtzke JF: Rating neurologic impairment in multiple sclerosis: an expanded disability status scale (EDSS). Neurology 1983;33: 1444-1452 (available on CD as: Kurtzke JF: EDSS bibliographic material. 21st Annual Meeting of the Consortium of MS Centers, Washington, May 30 to June 2, 2007, support@mscare.org).
16 Veterans Administration Multiple Sclerosis Study Group (Nagler B, chairman): Isoniazid in treatment of multiple sclerosis. Report of Veterans Administration Cooperative Study. JAMA 1957;163:168-172.

17 Kurtzke JF, Berlin L: The effects of isoniazid on patients with multiple sclerosis: preliminary report. Am Rev Tuberc 1954;70:577592.

18 Rose AS, Kuzma JW, Kurtzke JF, Sibley WA, Tourtellotte WW, in collaboration with Dixon WJ, Foley JM, Geschwind N, Kane CA, MacKay RP, McDowell FH, Scheinberg LC, Schumacher GA, White PT: Cooperative study in the evaluation of therapy in multiple sclerosis: ACTH vs placebo in acute exacerbations. Preliminary report. Neurology 1968;18:1-10, protocol 1-20 plus forms (22 pp).

19 Rose AS, Kuzma JW, Kurtzke JF, Namerow NS, Sibley WA, Tourtellotte WW, in collaboration with Dixon WJ, Foley JM, Geschwind N, MacKay RP, McDowell FH, Scheinberg LC, Schumacher GA, White PT, and with the assistance of Brown AJ, Fusillo MG, Johnson W, Kane CA, Lee JE, deOliviera MM, Rumberg J, Simpson JF: Cooperative study in multiple sclerosis: ACTH vs placebo. Final report. Neurology 1970;20:1-59.
20 Kurtzke JF: Risk factors, course, and prognosis of multiple sclerosis; in Cazzullo CL, Caputo D, Ghezzi A, Zaffaroni M (eds): Virology and Immunology of Multiple Sclerosis: Rationale for Therapy. Berlin, Springer, 1986, pp 349-357.

21 Kurtzke JF, Beebe GW, Nagler B, Kurland LT, Auth TL: Studies on the natural history of multiple sclerosis. 8. Early prognostic features of the later course of the illness. J Chron Dis 1977;30:819-830.

22 Kurtzke JF: Origin of DSS: to present the plan. Multiple Sclerosis 2007;13:120-123 (available on CD as: Kurtzke JF: EDSS bibliographic material. 21st Annual Meeting of the Consortium of MS Centers, Washington, May 30 to June 2, 2007, support@mscare. org).

23 Kurtzke JF: Further notes on disability evaluation in multiple sclerosis, with scale modifications. Neurology 1965;15:654-661. 